\title{
Education
}

\section{Taking advantage of the IRIS experience: utilizing the potential of trainees and faculty}

\section{Ljuba Bacharova ${ }^{1}$, Dominika Komarova, ${ }^{2}$ Tomas Hromadka ${ }^{3}$, Vaclav Bacovsky ${ }^{4}$, Peter Skrak ${ }^{5}$, Brigita Benkoova ${ }^{6}$,}

Allan Bohm ${ }^{2,7,8}$

${ }^{1}$ International Laser Center CVTI, Bratislava, Slovakia

2 Premedix Academy, Bratislava, Slovakia

${ }^{3}$ Institute of Neuroimmunology, Slovak Academy of Sciences, Bratislava, Slovak Republic

${ }^{4}$ Institute of Biophysics of the Czech Academy of Sciences, Brno, Czech Republic

${ }^{5}$ Pediatric Cardiac Center, National Institute of Cardiovascular Diseases, Bratislava, Slovakia

${ }^{6}$ Slovak Medical University, Bratislava, Slovak Republic

${ }^{7}$ National Institute of Cardiovascular Diseases, Bratislava, Slovak Republic

${ }^{8}$ University Hospital Bratislava, Faculty of Medicine, Comenius University, Bratislava, Slovak Republic

The International Research Interdisciplinary Schools (IRIS) have been providing training for young researcher for 15 years already (1). During these 15 years a solid experience was gained regarding the interactive and efficient methods of teaching, building international networks, stimulating and supporting international collaboration, exchanging information on training, scientific events, research projects, etc. Additional robust experience with on-line training has been added primarily due to the Covid-19 pandemic $(2,3)$. So far, 432 participants attended the courses, and 77 people were involved as faculty and/or organizers, many of the participants are now serving as active faculty members during training. This represents a highly valuable human potential for further dissemination of the IRIS initiative. The number of trainees in Slovakia has reached a critical number we wanted to benefit from. Here we summarize our experience with applying the IRIS approach to local / national seminars for young researchers in Slovakia.
The very first seminars on "Biomedical Research" were organized sporadically since 2017. In 2019 they were included into the portfolio of educational activities of Premedix Academy NGO (4), which now manages their organization as well as the IRIS courses. The program of the seminars followed the program of the IRIS, i.e. it consisted of the same series of workshops (Table 1). The program also used the same format for developing the Research Study Protocol. However, there was a difference in the sequence of the topics - the topic of the original workshop \#1 was discussed in detail as the last one, to provide participants more time to become familiar with the topic.

Because of the COVID-19 pandemic, the courses were conducted on-line, using the Zoom platform. The number of participants was limited, since they were working on their individual projects; a modification of the original IRIS arrangement where participants work in groups. The discussions were focused primarily on the development of study protocols.

\begin{tabular}{|l|l|}
\hline \multicolumn{2}{|l|}{ Table 1. The program of the course on biomedical research } \\
\hline Workshop 1 & Significance of the study and current knowledge \\
\hline Workshop 2 & Methods I: Study Population and Outcomes \\
\hline Workshop 3 & Methods II: Data Collection and Analysis \\
\hline Workshop 4 & Research Administration \\
\hline Presentation of completed protocols and discussion. \\
\hline
\end{tabular}

Address for correspondence: Ljuba Bacharova, International Laser Center CVTI, Ilkovicova 3 84104 Bratislava, Slovak Republic, email, bacharova@ilc.sk

Received: 30.03.2021 Revised: 21.04.2021 Accepted: 21.04.2021

Copyright @2021 Heart, Vessels and Transplantation doi: $10.24969 /$ hvt.2021.250 


\begin{tabular}{|c|c|}
\hline Day 1 & $\begin{array}{l}\text { The basics of academic writing: } \\
\text {-Formal structure and academic language } \\
\text {-A research study paper } \\
\text {-A review paper } \\
\text {-A well-developed paragraph, topic sentence }\end{array}$ \\
\hline Day 2 & $\begin{array}{l}\text { Writing an Abstract: structure, content. } \\
\text {-Discussion on selected participants' abstracts } \\
\text { Paraphrasing, summarizing / synthesizing }\end{array}$ \\
\hline Day 3 & $\begin{array}{l}\text { Writing Material and Methods/ Results: structure, content } \\
\text {-Discussion on selected participants' Methods/ Results part of their manuscripts. } \\
\text { Using tenses (present, past, present perfect, simple, continuous), using active - passive voices }\end{array}$ \\
\hline Day 4 & $\begin{array}{l}\text { Writing an Introduction: structure, content } \\
\text {-Discussion on selected participants' Introduction part of their manuscripts. } \\
\text { Cohesion, linking words }\end{array}$ \\
\hline Day 5 & $\begin{array}{l}\text { Writing a Discussion: structure, content } \\
\quad \text {-Discussion on selected participants' Discussion part of their manuscripts }\end{array}$ \\
\hline Day 6 & $\begin{array}{l}\text { Completing a draft manuscript } \\
\text { Communication with the editor / reviewers }\end{array}$ \\
\hline
\end{tabular}

Additionally, the discussions also stressed the interconnection between preparing own research protocols and understanding the relation between the preparation of a qualified research study protocol and the expected "outcome" of the research - the manuscript to be submitted to a scientific journal.

Two courses were organized from October 2020 to February 2021, a course on biomedical research and a course on academic writing. Their topics were interrelated, and some participants attended both of courses. As mentioned above, the participants were working and discussing their own individual projects/ manuscripts, so the number of participants was limited to six. The program of the course on academic writing is presented in Table 2 .

The courses were conducted by one facilitator and additional faculty was invited for discussions according to their availability, from Slovakia as well as from abroad, namely Sweden and the Czech Republic. English language was primarily used, in some cases we switched to Slovak to encourage more involvement.

Summarizing the main observations and experience from these pilot on-line courses:

-Young researchers are not accustomed to discuss, to provide and accept feedback. Discussion / questions are somehow perceived as a sort of an exam, as a testing of their knowledge, not as an expression of others' interest. This attitude was more seen during the first workshops, fortunately during the course was reduced.

-Questions frequently motivated participants to defend their views or positions, and not to explain their views, opinions, understanding, or interpretations. Participants were not accustomed to take notes from discussions for further consideration.

-Medical environment, and especially clinical one, is a hierarchical authority-based environment that does not motivate young researchers to express their opinion, and thus limits the discussion.

-The medical PhD students at the beginning of the PhD program (the first year) are more focused on gaining clinical experience and becoming familiar with the methodological technical aspects of the diagnostic / experimental methods;

-Physicians in clinical research have additional duties, such as full-time clinical practice, teaching, participation at different courses. These courses are predominantly discipline-specific, rarely focused on "soft" skills, "how to do research", or "academic writing".

-On the other hand, young researchers are under stress to publish, but paradoxically, writing is not perceived as an integral part either of their research or of their training. Procrastination is frequently seen as a solution, waiting for the "less busy" periods, which are actually quite rare. 
-Frequently, a problematic support from supervisors is mentioned. On the one hand there are complaints about the limited time devoted to students (5), on the other hand supervision includes some sorts of "local" modifications that deviate from or are not sufficiently consistent with the requirements of international scientific journals. This situation is even more pronounced during the Covid-19 pandemic, which is time demanding both for PhD students and their mentors. In spite of increasing interest among young researchers and their mentors, there is a considerable additional load observed especially in clinical workers, other health-care professionals, and volunteers.

-A considerable limitation is the English language. The university education in Slovakia is performed in the Slovak language. Although the level of English knowledge of students is increasing, the lack of practice in English is noticeable. At medical schools the stress is on the English medical terminology, and a little attention is devoted to the academic English and academic writing with its specific rules.

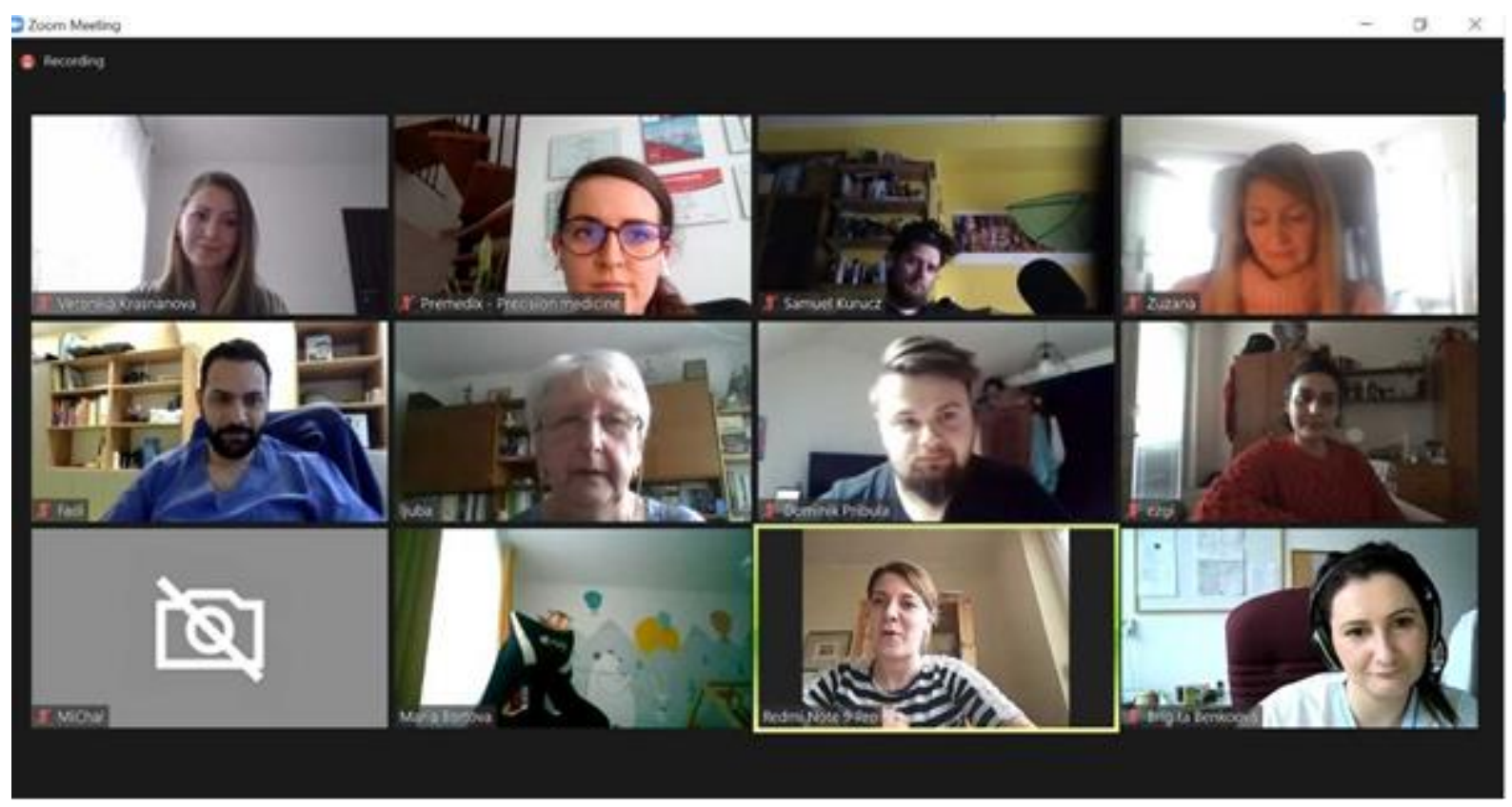

Figure 1. The on-line discussion

A specific experience was gained from the on-line format of the courses.

-The Zoom online platform appears as an useful tool for the communication during persistent pandemic situation to unable organizing different educational courses. The invaluable benefit of this platform is the video recording that was appreciated by course participants for self-study or playback, if they could not attend the course online. No matter how motivated the current limited situation is for using similar online approaches for communication, personal interaction between the lecturer (course mentor) and the participant seems to be an irreplaceable part of our courses.

-An important advantage compared to the classical in-person seminars is no need for travel arrangements for participants and the faculty, which considerably reduced the costs and time, and also extended the availability of the course. While originally the very first seminars were limited to Bratislava, now they can be conducted at national and also international levels, which contributes to additional building of national and international networks.

-Timing of courses was a specific issue. Since the on-line format enables participation of foreign participants and faculty, it requires a careful consideration of time zones. The optimal timing for the local participants and faculty was late afternoon, i.e. after the working hours. In our courses we had participants from countries where the time zone was one hour ahead, which did not represent a problem. 
-An important benefit was the gained experience with on-line tools, which otherwise would not be that urgent. An optimal combination of on-line and in-person formats represents a great potential for the future.

-On the other hand, the on-line format is quite demanding on the ability of participants to concentrate for longer periods of time. Nonverbal communication and visual contact are limited, nearly absent, which also contributes to decreasing concentration. And, sometimes, the distractions from the domestic, but also working environment cannot be completely avoided.

We would like to open a discussion on this topic - how to teach young researcher the soft skills required for doing research, as well as how to combine the on-line and in-person formats in an optimal way. We would appreciate if you could share your experience as organizers as well as participants.
Peer-review: Internal and external Conflict of interest: None to declare Authorship: L.B., D.K., T.H., V.B., P.S., B.B., A.B. Acknowledgement and funding: None to declare

\section{References}

1.Bacharova L, Eisenstein E, Kudaiberdieva G, Lipton J, Sorokivskyy M, Bang LE, et al. IRIS Summary Report 2006-2020; Premedix Academy 2021, Bratislava.

2.Bacharova L, Komarova D, Hromadka T, Bohm A. IRIS 2020: moving to the virtual space: IRIS Slovakia 2020 digital - the first step to the on-line arrangement. Heart, Vessels and Transplantation 2020; 4: 170-6.

3.Bacharova L, Eisenstein E, Lipton J, Pidvalna U, Abibillaev D, Tiumkin P, et al. IRIS 2020: moving to the fully virtual space: IRIS Kyrgyzstan 2020 digital - the first full on-line IRIS course. Heart, Vessels and Transplantation 2020; 4: 177-82.

4.https://academy.premedix.org/IRIS/

5.MESA10 Survey "To dá rozum", 2018. Available at: URL: https://analyza.todarozum.sk

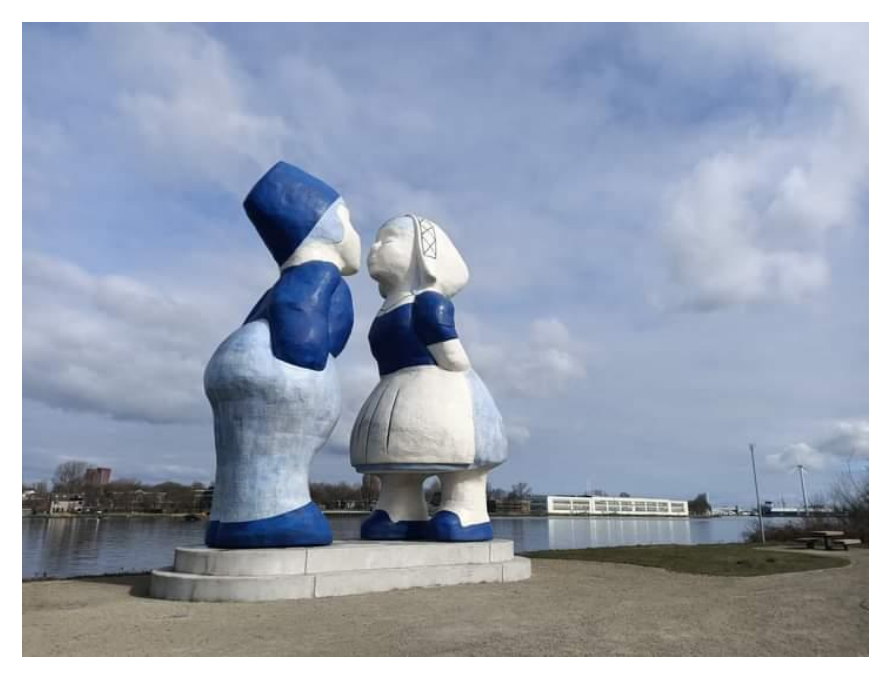

The Delft Blue Kissing Couple XXXL, Hempontplein, The Nothern Sea Canal, Amsterdam, The Netherlands. Jonathan Lipton, February 2021. Tasmania, Australia.

(The statue was built in 2017 along Bicycle route to stimulate people to come to the area bicycle. Statue was made in collaboration by Saske van der Eerden, ArchitecturePlus and Van Zuilen Construction Advice. ) 